\title{
Infrared skin damage thresholds from 1940-nm continuous-wave laser exposures
}

Jeffrey W. Oliver

Air Force Research Laboratory

2624 Louis Bauer Drive

San Antonio, Texas 78235-5148

\author{
David J. Stolarski \\ Gary D. Noojin \\ Harvey M. Hodnett \\ Corey A. Harbert \\ Kurt J. Schuster \\ Michael F. Foltz \\ TASC Incorporated \\ 4241 Woodcock Drive, Suite B-100 \\ San Antonio, Texas 78228
}

\section{Semih S. Kumru}

Air Force Research Laboratory

2624 Louis Bauer Drive

San Antonio, Texas 78235-5148

\section{Clarence P. Cain}

TASC Incorporated

4241 Woodcock Drive, Suite B-100

San Antonio, Texas 78228

\section{J. Finkeldei}

Gavin D. Buffington

Fort Hays State University

Department of Physics

600 Park Street

Hays, Kansas 67601-4099

\section{Isaac D. Noojin}

TASC Incorporated

4241 Woodcock Drive, Suite B-100

San Antonio, Texas 78228

\section{Robert J. Thomas}

Air Force Research Laboratory

2624 Louis Bauer Drive

San Antonio, Texas 78235-5148

\begin{abstract}
A series of experiments are conducted in vivo using Yucatan mini-pigs (Sus scrofa domestica) to determine thermal damage thresholds to the skin from 1940-nm continuous-wave thulium fiber laser irradiation. Experiments employ exposure durations from $10 \mathrm{~ms}$ to $10 \mathrm{~s}$ and beam diameters of approximately 4.8 to $18 \mathrm{~mm}$. Thermal imagery data provide a time-dependent surface temperature response from the laser. A damage endpoint of minimally visible effect is employed to determine threshold for damage at 1 and $24 \mathrm{~h}$ postexposure. Predicted thermal response and damage thresholds are compared with a numerical model of optical-thermal interaction. Results are compared with current exposure limits for laser safety. It is concluded that exposure limits should be based on data representative of large-beam exposures, where effects of radial diffusion are minimized for longer-duration damage thresholds. (C) 2010 Society of Photo-Optical Instrumentation Engineers. [DOI: 10.1117/1.3523622]
\end{abstract}

Keywords: infrared laser; skin damage; exposure limits; laser-thermal tissue response. Paper 10406R received Jul. 20, 2010; revised manuscript received Oct. 20, 2010; accepted for publication Oct. 22, 2010; published online Dec. 16, 2010.

\section{Introduction}

Standardscommittees are currently pursuing revisions of laser safety standards. Revisions are focused on the near-infrared region and are based primarily on new data collected within the wavelength region of 1100 to $1400 \mathrm{~nm} .{ }^{1}$ However, laser systems operating near the wavelength of $2000 \mathrm{~nm}$ are becoming relatively widespread in industrial, medical, and research markets.

Address all correspondence to: David J. Stolarski, TASC, 2624 Louis Bauer Drive, Brooks City, Texas 78265-5128. Tel: 210-536-4796; Fax: 210-534-0420. E-mail: david.stolarski.ctr@brooks.af.mil.

\begin{abstract}
Many laser systems employ high-power Ho:YAG and thulium fiber lasers. These lasers are being used in expanding ranges of application and may, in some cases, effectively replace $\mathrm{CO}_{2}$ lasers. As exposure limits for these near-2000-nm wavelengths were based on limited data, we have undertaken a series of experiments to add information for the current revision of safety standards. These will ensure that the use of new systems have appropriately validated exposure limits, based on experimentally observed and theoretically predicted mechanisms.
\end{abstract}

$1083-3668 / 2010 / 15(6) / 065008 / 10 / \$ 25.00$ C 2010 SPIE 
In a previous study, ${ }^{2-4}$ the results of experiments and tests on Yucatan mini-pigs were presented for a 2000-nm thulium fiber laser for various exposure durations and beam diameters applied to skin. In that study, experimental conditions were limited in scope due to available laser power. Here, parameters for mini-pig skin exposures using a similar but more powerful 1940-nm laser were selected to bracket the parameters used in the earlier study. This allows for conditions where damage can be produced in a shorter time and with a larger maximum beam diameter delivered to the target. The Yucatan mini-pig has been established as a model for human skin damage studies due to the morphological and physiological similarities to human skin. ${ }^{5}$

Thermally induced damage to laser irradiated skin depends on the time-temperature response of tissues. This begins with the local absorption of the laser energy that heats the tissue. The tissue can be affected by this heating through denaturation of cellular proteins, leading to a subsequent apoptosis or overt necrosis. At higher temperatures, thermal coagulation, collagen hyalinization, and changes in optical properties such as increased scattering or birefringence can be observed. , $^{6,7}$

Several authors have contributed to numerical simulations of optical-thermal response of tissues and models of the damage process. These are largely built on the original works of Mainster et al., ${ }^{8}$ and Takata, Zaneveld, and Richter, ${ }^{9}$ with many authors contributing through the addition of increased fidelity. $6,10,11$ Models that represent the skin as a two- or three-layer construct, with a Beer's law absorption term for the laser energy deposition, have been shown to accurately predict the opticalthermal response of the tissue. . $^{3,1}, 12$ Thermal diffusion solutions are most often computed through finite element or finite difference methods. Increased accuracy has been demonstrated when temperature-dependent surface cooling associated with the evaporation of water is included. ${ }^{13}$ In the comprehensive analysis of Chen et al., ${ }^{4}$ thermal damage was shown to most accurately mimic histology for an Arrhenius model when based on the parameters presented by Gaylor. ${ }^{14}$ The extent of damage was to a depth of 200 to $300 \mu \mathrm{m}$ at the observed minimal visible lesion threshold. When the criterion of damage to this depth is applied to modeling, the predicted damage thresholds accurately follow the minimally visible lesion endpoint thresholds. ${ }^{4}$

The study of thermal injury under an expanded range of exposure parameters allows for the formulation of broadly applicable trends from which exposure limits can be defined. In addition, the regions in which experiments diverge from theory may establish transition regions for mechanisms of damage. From this supposition, one can derive corresponding trends in empirical descriptions for exposure limits for the skin as well as the eye, provided similar data are available from ex-vivo studies, for example, damage to the retina. ${ }^{15}$

\section{Methods}

\subsection{Experiment}

The animal use protocols for this research were approved by Institutional Animal Care and Use Committees at Brooks CityBase, Texas. Animals involved in this study were procured, maintained, and used in accordance with the (United States Department of Agriculture) USDA Animal Welfare Act and Animal Welfare Regulations, and the National Research Council Guide for the Care and Use of Laboratory Animals.
A total of 12 Yucatan mini-pigs ( 30 to $50 \mathrm{~kg}$ ) were used for this study. All procedures were performed during the light cycle of the animal's normal schedule. Subjects had food withheld for $12 \mathrm{~h}$ prior to the procedure. Anesthesia was initiated with intramuscular (IM) Telazol (4 mg/kg). Animals were then orotracheally intubated and anesthesia was maintained with isoflurane (1 to $3 \%)$ throughout the procedure. Heart rate, oxygen saturation $\left(\mathrm{SpO}_{2}\right)$, and respiration were monitored throughout the procedure. After animals were stabilized under anesthesia, hair was removed from the flank using electric clippers, followed by a five minute application of a depilatory (Nair ${ }^{\mathrm{TM}}$ ). The depilatory was discontinued after two initial subjects due to persistent irritation. The flank was cleaned with warm soap and water, rinsed again with warm water, and dried with a lint-free disposable cloth. Core body temperature of animals was maintained using a $\mathrm{T} / \mathrm{Pump}^{\mathrm{TM}}$ circulating water blanket (Gaymar Incorporated, Orchard Park, New York). A grid pattern was drawn on the flank of the pigs with a black marker, with grid spacing of $1.5,2$, or $3 \mathrm{~cm}$, depending on the planned laser parameters for the exposure. Skin biopsy punches of 6 to $8 \mathrm{~mm}$ in diameter were collected from select exposure sites at either 1 or $24 \mathrm{~h}$ postexposure. At the end of the procedure, buprenorphine was administered IM as an analgesic. All animals were reanesthetized postlaser exposure for a 24-h follow-up lesion assessment. After laser exposure to the first flank, animals were allowed to recover for at least three weeks (range of three weeks to four months) before the other flank was exposed. After the second flank was exposed and evaluated at $24 \mathrm{~h}$, the animals were released from the protocol.

For this skin study, a fiber laser system was employed as the source for animal exposures, as shown in the schematic diagram of Fig. 1. An IPG Photonics (Oxford, Massachusetts) model TLR-150-1940A thulium laser with a maximum specified power of $150 \mathrm{~W}$ was integrated into the optical system. Laser emission was electronically gated through the IPG programmable control interface. The output from the fiber was quasi-Gaussian with a $1 / e^{2}$ diameter of approximately $0.5 \mathrm{~cm}$. The size of the beam delivered to the target was controlled via a beam-expanding telescope (BET). Due to variability of the spatial distribution of the beam with internal power settings, the laser was operated at a fixed power within $30 \%$ of maximum output. Therefore, combinations of beamsplitters were inserted in the beam as required to adjust total energy to target. For each laser exposure, reference energy from a low-power sample of the beam was recorded with a PM10 power detector (Coherent Incorporated, Santa Clara, California). Measurements acquired with the PM10 in the low-power reference beam were compared with measurements obtained with a PM150 detector (Coherent Incorporated, Santa Clara, California) placed near the target plane before testing, at the scheduled midway time point during testing, and after each sequence of exposures, to assure the integrity of the high-energy portion of the beam train. This also confirmed the accurate quantification of energy delivered to the target.

Beam diameters of $0.48,1.0$, and $1.8 \mathrm{~cm}\left(1 / e^{2}\right)$ with quasiGaussian profiles were used in this study. Beam size at the target was measured with a Pyrocam III (Spiricon, Logan, Utah) or 7290A extended range camera (Electro Physics, Fairfield, New Jersey) at the time of setup for each beam configuration. Output from the telescopes was collimated to maintain spot size over the 


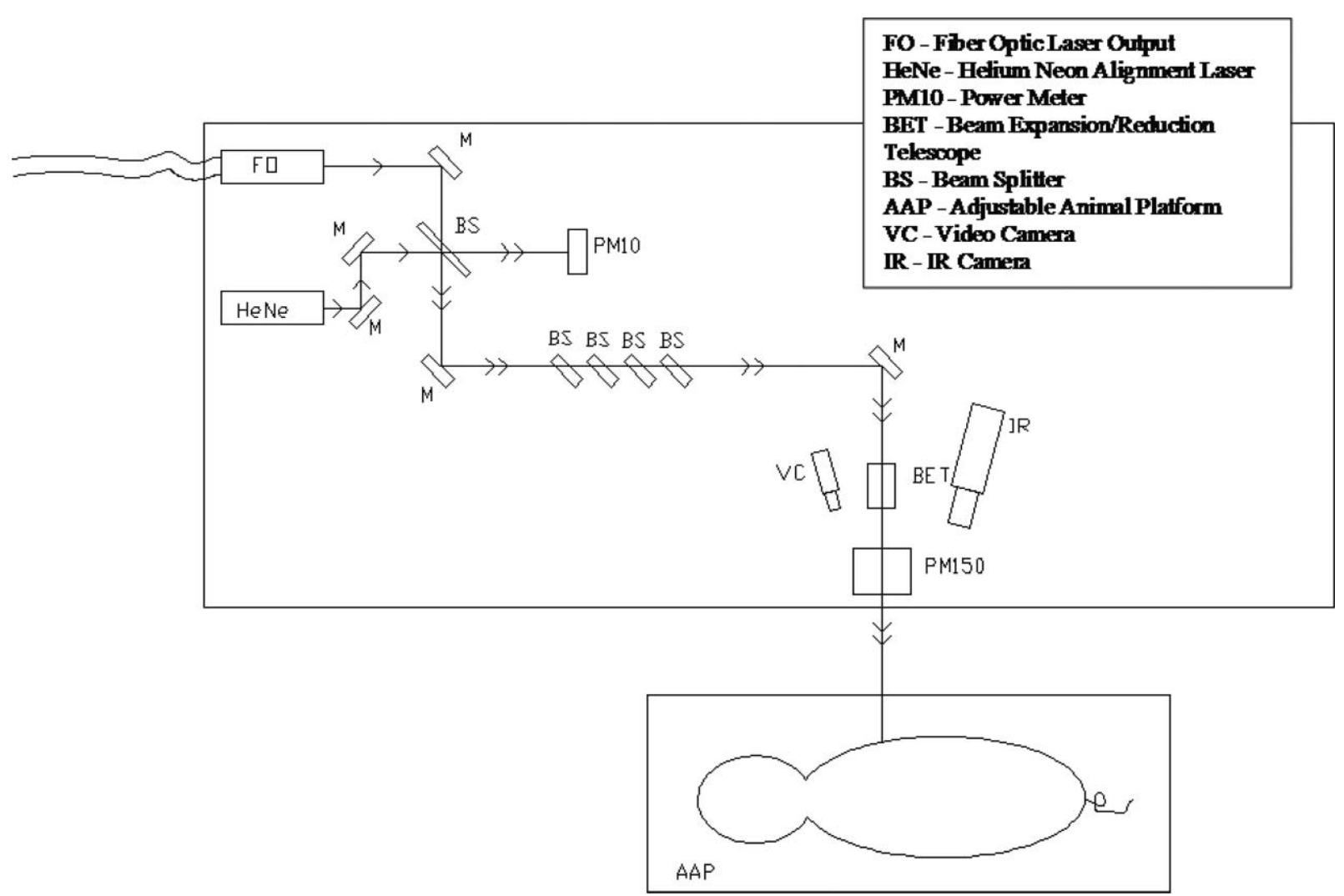

Fig. 1 Experimental configuration for 1940-nm laser skin exposures.

expected range of target distance determined from the anatomical confirmation of the animals. Consistency of the beam size was verified during testing with laser alignment paper (ZAP$\mathrm{IT}^{\mathrm{TM}}$, Kentek Corporation, Pittsfield, New Hampshire). Laser on-times were verified at the time of setup using a Coherent J4-09 pyroelectric joule probe (Coherent Incorporated, Santa Clara, California) coupled to a TDS 3054B digital oscilloscope (Tektronix, Incorporated, Beaverton, Oregon). The beam diameter and profile was also confirmed by examining the imagery of thermal response at early time points $(100 \mathrm{~ms})$ after the laser on-time in the experiment.

The 1940-nm laser beam was coaligned with a helium neon (HeNe) laser (Meredith Instruments, Glendale, Arizona) to facilitate beam placement at the target. A model DXC-107A (Sony, San Diego, California) Iris CCD color video camera (VC) was also utilized in this arrangement for target position verification. This video camera was mounted on a custom translation stage and was cofocused at a common region of interest in the target plane with a SC4000 (IR) InSb high-framerate thermal camera (FLIR Systems, Boston, Massachusetts), which is sensitive to radiation in the 3- to 5- $\mu \mathrm{m}$ wavelength thermal emission band. This camera sensitivity was specifically selected to be out of band for the lasers under test, so that surface temperature changes during laser exposure could be obtained without backscatter from the target distorting the measurements. The thermal camera was synchronized with the control electronics for the laser to trigger data collection to begin at greater than or equal to $100 \mathrm{~ms}$ prior to laser exposure of the skin. The camera was programmed to collect data for a period of time between 6 and $30 \mathrm{~s}$ beyond the completion of each laser exposure to capture the trajectory of thermal decay of the laser-heated tissue. Fine adjustments in IR camera focus were made prior to each laser exposure using an NTSC display of the thermal image of emissivity contrast sources originating from inhomogeneities in skin structure, such as wrinkles or hair follicles.

Anesthetized pigs were placed in a customized pig sling $(\mathrm{BH}$, Incorporated, Wheatland, Wyoming), which was mounted on rails and motorized for rapid and repeatable horizontal position adjustment of the test subjects. A hand crank on the sling was used to adjust the vertical position of the subject prior to each laser exposure. In this manner, each area of the grid that had been drawn on the flank of the pig was sequentially addressed with a laser exposure, according to a predetermined randomized selection of energy levels distributed over a range encompassing the anticipated threshold level. Energy for each laser exposure and any remarkable observations were recorded immediately following each exposure.

The Probit procedure ${ }^{16}$ was used to estimate the dose for creating a minimal visual lesion (MVL) for each laser configuration used in the skin studies (i.e., $\mathrm{ED}_{50}$ ). Each exposure site was assessed as positive or negative for damage, by three experienced observers, with at least two of three in agreement constituting a positive reading. A minimum of three animals was used for each laser configuration investigated. In addition, 95\% confidence intervals were calculated from the data. An adequate number of data points was collected to ensure that the magnitude of upper and lower fiducial limits at the $\mathrm{ED}_{50}$ level varied no more than $50 \%$. Furthermore, a Probit-curve slope greater than two, with aggregated 24-h observation data, was set as a minimal experimental ending point for the study of a 
specific exposure condition. Here, the slope reported represents the first derivative (probability per dose) of the dose-response curve at the $\mathrm{ED}_{50}$ point generated from the Probit analysis method.

\subsection{Models}

A numerical model was developed to simulate laser energy deposition, heat transfer, and a prediction of the tissue damage threshold. To simulate the experimental data presented here, the model is essentially a 2-D (cylindrical coordinate), finite difference solution to the Pennes bio-heat equation, given by Eq. (1).

$\rho c \frac{\partial v}{\partial t}=\frac{\kappa}{r} \frac{\partial v}{\partial r}+\frac{\partial}{\partial r}\left(\kappa \frac{\partial v}{\partial r}\right)+\frac{\partial}{\partial z}\left(\kappa \frac{\partial v}{\partial z}\right)+A(r, z, t)+q$.

In Eq. (1), $v$ is the local temperature rise at coordinates $(r, z)$, $\kappa$ is the thermal conductivity, $\rho$ is the local tissue volumetric density, $c$ is the specific heat, and $q$ is the power density loss or gain due to blood flow. The source term $A$ is configured as a Gaussian lateral distribution, and assumes an energy deposition rate proportional to the local irradiance, as shown in Eq. (2).

$$
A(r, z, t)=\exp \left(\frac{-r^{2}}{a^{2}}\right) H_{0}(\lambda, t) \mu_{a}(z, \lambda) .
$$

Here, $\mu_{a}$ is the local linear absorption coefficient in the tissue, $a$ is the beam radius, and $H_{0}$ is the incident peak irradiance after any surface reflective loss. Peak values are calculated as the total power divided by the $1 / e$ area. The model includes representations of surface boundary conditions mimicking the loss of energy to the surrounding environment. ${ }^{13}$

The resultant time dependence of temperature is evaluated with an Arrhenius damage model. ${ }^{6}$ A damage threshold was determined by searching for a laser power that produced a prescribed value damage integral at $250 \mu \mathrm{m}$ depth in the tissue structure, corresponding to prior assessments of histology for visually observed damage endpoints. ${ }^{4}$ The Arrhenius damage integral is given by Eq. (3), in which $R$ is the universal gas constant, $C$ is the normalization rate constant (molecular collision frequency factor), and $E$ is the activation energy. The value is computed at each $(z, r)$ coordinate in the model, based on the temperature history $T=T(z, r, t)$ for that coordinate. A value of $\Omega=1$ is assumed to represent the threshold for damage. Physical constants and other values for Eqs. (1), (2), and (3) are provided in Table 1.

$$
\Omega(r, z)=C \int_{t_{1}}^{t_{2}} \exp \left(\frac{-E}{R T}\right) d t .
$$

The tissue geometry was represented within the model as a three-layer structure consisting of epidermis and dermis skin layers each having homogeneous properties. A third layer of tissue was added below the dermis to simulate the insulating effect of fatty tissue. Although this layer does not absorb the laser energy directly, the layer was found to affect predicted outcomes for longer exposure times, where significant thermal diffusion occurred in regions beyond the dermis during the laser exposure time. Parameters for the fat layer were obtained from
Table 1 Tissue parameters used in simulation of heat transfer and

\begin{tabular}{|c|c|c|}
\hline Skin layer thickness & Epidermis & 0.0068 \\
\hline \multirow[t]{2}{*}[\mathrm{cm}]{} & Dermis & 0.1432 \\
\hline & Fatty tissue & Infinite \\
\hline Absorption coefficient & Epidermis & 33.1 \\
\hline \multirow[t]{2}{*}[1/\mathrm{cm}]{} & Dermis & 88.3 \\
\hline & Fatty tissue & 0.5 \\
\hline Density, $\rho$ & Epidermis & 1.21 \\
\hline \multirow[t]{2}{*}[\mathrm{g}/\mathrm{cm}^{3}]{} & Dermis & 1.06 \\
\hline & Fatty tissue & 0.85 \\
\hline Specific heat, c & Epidermis & 2.244 \\
\hline \multirow[t]{2}{*}[\mathrm{J}/\mathrm{g}^{\circ}\mathrm{C}]{} & Dermis & 3.663 \\
\hline & Fatty tissue & 2.07 \\
\hline Conductivity, $\kappa$ & Epidermis & 0.002 \\
\hline \multirow[t]{2}{*}[\mathrm{W}/\mathrm{cm}^{\circ}\mathrm{C}]{} & Dermis & 0.0049 \\
\hline & Fatty tissue & 0.0016 \\
\hline Convective heat transfer rate & Epidermis & 0.0015 \\
\hline \multirow[t]{2}{*}[\mathrm{W}/{}^{\circ}\mathrm{C}\mathrm{cm}^{2}]{} & Dermis & $\mathrm{N} / \mathrm{A}$ \\
\hline & Fatty tissue & $N / A$ \\
\hline \multirow[t]{3}{*}{ Emissivity } & Epidermis & 0.80 \\
\hline & Dermis & $\mathrm{N} / \mathrm{A}$ \\
\hline & Fatty tissue & $N / A$ \\
\hline Blood flow rate & Epidermis & 0.0 \\
\hline \multirow[t]{2}{*}[\mathrm{g}/\mathrm{cm}^{3}\mathrm{s}]{} & Dermis & 0.003 \\
\hline & Fatty tissue & 0.0 \\
\hline Molecular collision frequency factor, C & Epidermis & $3.10 \times 10^{98}$ \\
\hline \multirow[t]{2}{*}[1/\mathrm{s}]{} & Dermis & $3.10 \times 10^{98}$ \\
\hline & Fatty tissue & $N / A$ \\
\hline Denaturation activation energy, $\mathrm{E}$ & Epidermis & $6.28 \times 10^{5}$ \\
\hline \multirow[t]{2}{*}[\mathrm{J}/\mathrm{mole}]{} & Dermis & $6.28 \times 10^{5}$ \\
\hline & Fatty tissue & N/A \\
\hline
\end{tabular}
damage thresholds. ${ }^{3,9,12}$ Rate process values are from Henriques. ${ }^{22}$

recent simulations of laser tissue welding. ${ }^{12}$ Thermal and optical properties of the skin layers were estimated using equations derived by Takata, Zaneveld, and Richter ${ }^{9}$ with the assumption of $80 \%$ water content in the dermis and $30 \%$ in epidermis, as did Chen et al. $^{3}$ The absorption coefficient does change fairly rapidly between 1940 and $2000 \mathrm{~nm}$. This difference was programmed 
Oliver et al.: Infrared skin damage thresholds from 1940-nm continuous-wave laser exposures

Table 2 Summary of $\mathrm{ED}_{50}$ one-hour damage threshold data obtained for skin damage with a 1940-nm laser. Irradiance and radiant exposure values are peak values within the quasi-Gaussian profile.

\begin{tabular}{|c|c|c|c|c|c|c|}
\hline $\begin{array}{l}\text { Number of } \\
\text { exposures }\end{array}$ & $\begin{array}{l}\text { Diameter } \\
{[\mathrm{cm}]}\end{array}$ & Time & $\begin{array}{c}\mathrm{ED}_{50}(95 \% \text { conf inter }) \\
{[\mathrm{J}]}\end{array}$ & $\begin{array}{l}\text { Probit } \\
\text { slope }\end{array}$ & $\begin{array}{l}\text { Irradiance } \\
{\left[\mathrm{W} / \mathrm{cm}^{2}\right]}\end{array}$ & $\begin{array}{c}\text { Radiant exposure } \\
{\left[\mathrm{J} / \mathrm{cm}^{2}\right]}\end{array}$ \\
\hline 122 & 0.48 & $10 \mathrm{~ms}$ & $0.244(0.183$ to 0.301$)$ & 3.45 & 269 & 2.69 \\
\hline 93 & 0.48 & $70 \mathrm{~ms}$ & $1.03(0.892$ to 1.13$)$ & 10.2 & 162 & 11.4 \\
\hline 144 & 0.48 & $10 \mathrm{~s}$ & 2.10 (1.86 to 2.35$)$ & 14.5 & 2.31 & 23.1 \\
\hline 144 & 1.0 & $50 \mathrm{~ms}$ & 1.49 (1.36 to 1.63$)$ & 7.37 & 75.9 & 3.79 \\
\hline 100 & 1.8 & $70 \mathrm{~ms}$ & 4.75 (4.35 to 5.24$)$ & 8.52 & 53.3 & 3.73 \\
\hline 77 & 1.8 & $10 \mathrm{~s}$ & $15.6(14.1$ to 17.0$)$ & 16.9 & 1.23 & 12.3 \\
\hline
\end{tabular}

into the model for comparison of the two studies. The model was run for laser exposure times between $10 \mathrm{~ms}$ and $10 \mathrm{~s}$ for beam diameters from 0.1 to $3 \mathrm{~cm}$. Simulation time included the cool-down phase after the pulse, sufficient to reach less than 1/e of the peak temperature achieved (one decay time constant).

\section{Results}

\subsection{Experimental Results}

A total of 680 skin exposures were completed on both flanks of 12 Yucatan mini-pigs with the 1940-nm laser. Five different configurations of laser parameters were utilized. The results of these experiments are summarized in Tables 2 and 3. Beam diameters reported are at $1 / e^{2}$ amplitude within the quasi-Gaussian distribution. Irradiance and radiant exposure values are computed as the peak value within the beam, rather than average values. This was done for comparison with the simulations where the beam is assumed to have infinite radial extent. Peak irradiance values also provide for a direct comparison to exposure limits.

Lesions generated with the 1940-nm laser were observed grossly as mild erythema and did not vary appreciably in appearance with laser parameters. Threshold damage observed with 10 -ms exposures was not distinct from threshold lesions formed with 10-s exposures. This observation indicates that there is not a transition in surface damage processes as a function of exposure duration at this wavelength of laser. Superthreshold damage presented as a second-degree burn characterized with blisters, which were acutely apparent. Further histological comparison of threshold lesions will be helpful in making the determination that there was no transition in primary damage processes at thresholds across the laser parameters tested.

Histology sections of Yucatan miniature pig skin punch biopsy samples were prepared with hematoxylin and eosin (HE) staining. Punch biopsies were collected from the central grid area to coincide with the center of the laser exposure site. The results of these studies have not returned from pathology, and their analysis will be presented at a later date.

\subsection{Modeling Results}

Important to the validation of models that predict tissue damage thresholds from dosimetry, is the intermediate confirmation that laser absorption and heat transfer are accurately simulated. To this end, computer simulations of the thermal response to the laser were conducted and compared with experimental temperatures from high-speed thermal imagery at

Table 3 Summary of ED 50 24-h damage threshold data obtained for skin damage with a 1940-nm laser. Irradiance and radiant exposure values are peak values within the quasi-Gaussian profile.

\begin{tabular}{|c|c|c|c|c|c|c|}
\hline $\begin{array}{l}\text { Number of } \\
\text { exposures }\end{array}$ & $\begin{array}{l}\text { Diameter } \\
{[\mathrm{cm}]}\end{array}$ & Time & $\begin{array}{c}\mathrm{ED}_{50}(95 \% \text { conf inter }) \\
{[\mathrm{J}]}\end{array}$ & $\begin{array}{l}\text { Probit } \\
\text { slope }\end{array}$ & $\begin{array}{l}\text { Irradiance } \\
{\left[\mathrm{W} / \mathrm{cm}^{2}\right]}\end{array}$ & $\begin{array}{l}\text { Radiant exposure } \\
{\left[\mathrm{J} / \mathrm{cm}^{2}\right]}\end{array}$ \\
\hline 122 & 0.48 & $10 \mathrm{~ms}$ & $0.201(0.172$ to 0.223$)$ & 6.51 & 221 & 2.21 \\
\hline 93 & 0.48 & $70 \mathrm{~ms}$ & 0.817 (no overlap) & - & 129 & 9.00 \\
\hline 144 & 0.48 & $10 \mathrm{~s}$ & 2.02 (1.75 to 2.33 ) & 16.4 & 2.23 & 22.3 \\
\hline 144 & 1.0 & $50 \mathrm{~ms}$ & $1.16(0.945$ to 1.30$)$ & 6.5 & 59.1 & 2.95 \\
\hline 100 & 1.8 & $70 \mathrm{~ms}$ & 3.73 (3.33 to 4.10$)$ & 7.64 & 41.9 & 2.93 \\
\hline 77 & 1.8 & $10 \mathrm{~s}$ & $5.72(5.36$ to 6.08$)$ & 10.4 & 1.27 & 12.7 \\
\hline
\end{tabular}




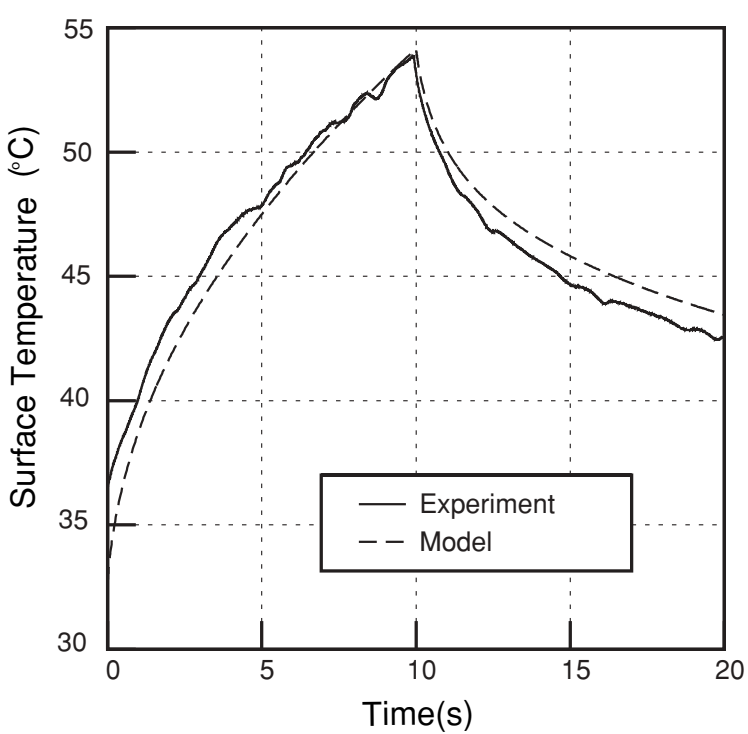

Fig. 2 Surface temperature response from a $1.8-\mathrm{cm}\left(1 / e^{2}\right)$ diameter beam compared to the numerical model prediction. Power was $1.65 \mathrm{~W}$, near the observed damage threshold of $1.3 \mathrm{~W}$.

the skin surface. Figure 2 demonstrates the extracted temperature near the centroid of an $1.8-\mathrm{cm}$ laser beam for a 10 -s exposure. Along with the experimentally recorded data is the result of a simulation of the surface temperature from the computer model. While we do see variability in experimentally measured surface temperature response, we find that the model accurately represents measured surface temperatures to within about $5^{\circ} \mathrm{C}$.

Figure 3 illustrates example compilations of peak temperature (at the end time of the laser exposure and near the laser centroid) as a function of laser power. Surface temperature was extracted from thermal imagery data and represents the maximum temperature within the exposed region, as averaged over a 20-pixel (approximately $400-\mu \mathrm{m}$ diam) region of interest. Power ranges selected for the simulation are representative of experimental conditions and spanned the measured damage thresholds. The figure illustrates the shot-to-shot experimental variability in surface thermal response. We note that the damage threshold consistently lies at approximately $60{ }^{\circ} \mathrm{C}$.

Model estimates of damage thresholds are compiled in Table 4 and Fig. 4. These estimates are based on the Arrhenius rate-process integral reaching a value of 1.0 at $250 \mu \mathrm{m}$ depth within the tissue simulation. The model consistently predicts the observed thresholds, with an average variation of about $30 \%$. This value can be compared with the average Probit fiducial limit (confidence interval) separation from the threshold value of approximately $10 \%$ with an additional uncertainty in measured irradiance (or radiant exposure) of approximately $20 \%$. In addition to the outcomes from the model presented here, Table 4 includes a comparison with an empirical model presented by Chen et al. ${ }^{2}$ for 2000-nm skin exposures. The model predicts a damage threshold as a function of beam diameter given by Eq. (4), where $t$ is the exposure time and $d$ is the beam diameter. The model is a result of a nonlinear least-squares fit to data collected between 0.25 - and 2.5-s exposure dura- tion, and is based on the empirical descriptions of Stoll and Greene. ${ }^{17}$

$$
E_{t h}=(5.669-1.81 d) t^{-0.794}\left(\mathrm{~W} / \mathrm{cm}^{2}\right) .
$$

\section{Discussion}

The primary goal of this study was to examine laser damage thresholds to skin for wavelengths near $2000 \mathrm{~nm}$, and compare these thresholds with current exposure limit definitions for a wide variety of exposure times. A numerical model, representative of experimental findings, allows for parametric analysis and estimation of damage thresholds relative to existing and proposed exposure limits. As experimental data are limited to sources currently available, the model allows for an investigation of several key issues regarding broader suitability of existing (or proposed) exposure limits.

The first issue is the effect of beam diameter on damage threshold. The heat equation [Eq. (1)] dictates that the rate of diffusion is influenced by the local temperature gradient. Tissue heated by a smaller diameter beam will experience a more rapid radial diffusion of energy and, for the same peak irradiance of exposure and of equal exposure time, will reach a lower temperature than that of a larger beam. From this analysis, one would expect that an infinitely wide beam would experience no radial diffusion loss and reach the upper limit for temperature for a given irradiance and time. Consequently, this infinitely wide beam would provide the lower bound for damage threshold. This 1-D (infinite in radial coordinate) limit can be simulated through modeling and be used to determine how closely experimental data approximate the anticipated lower bound for damage.

Figure 4 provides a comparison of modeled damage thresholds to experimental data from this study and the prior study of Chen et al. ${ }^{2}$ The 1940-nm data shown as closed circles bracket the exposure times from Chen et al.'s 2000-nm data. The 2000$\mathrm{nm}$ data from lowest to highest threshold for each exposure time were collected at 1.465-, 0.965-, and 0.483-cm 1/ $e^{2}$ Gaussian beam diameters. The filled circular points are from the data presented in Table 3, with data from lowest to highest (for a given time) threshold being the 1.8 - and $0.48-\mathrm{cm}$-diam conditions, respectively. The model results show (lower curves, for infinite beam diameter limit) the small difference in thresholds

Table 4 Summary of $\mathrm{ED}_{50}$ 24-h damage threshold data compared with model predictions of damage thresholds.

\begin{tabular}{cccccc}
\hline $\begin{array}{c}\text { Diameter } \\
{[\mathrm{cm}]}\end{array}$ & Time & $\begin{array}{c}\mathrm{ED}_{50} \\
{[\mathrm{~W}]}\end{array}$ & $\begin{array}{c}\text { Model } \\
{[\mathrm{W}]}\end{array}$ & $\begin{array}{c}\text { Percent } \\
\text { difference }\end{array}$ & $\begin{array}{c}\text { Chen et al. } \\
\text { prediction [W] }\end{array}$ \\
\hline 0.48 & $10 \mathrm{~ms}$ & 20.1 & 27.7 & +37 & 33.6 \\
0.48 & $70 \mathrm{~ms}$ & 11.7 & 4.41 & -62 & 7.18 \\
0.48 & $10 \mathrm{~s}$ & 0.202 & 0.14 & -30 & 0.14 \\
1.0 & $50 \mathrm{~ms}$ & 23.2 & 26.0 & +12 & 32.7 \\
1.8 & $70 \mathrm{~ms}$ & 53.3 & 60.9 & +14 & 50.7 \\
1.8 & $10 \mathrm{~s}$ & 1.62 & 1.28 & -24 & 0.986 \\
\hline
\end{tabular}



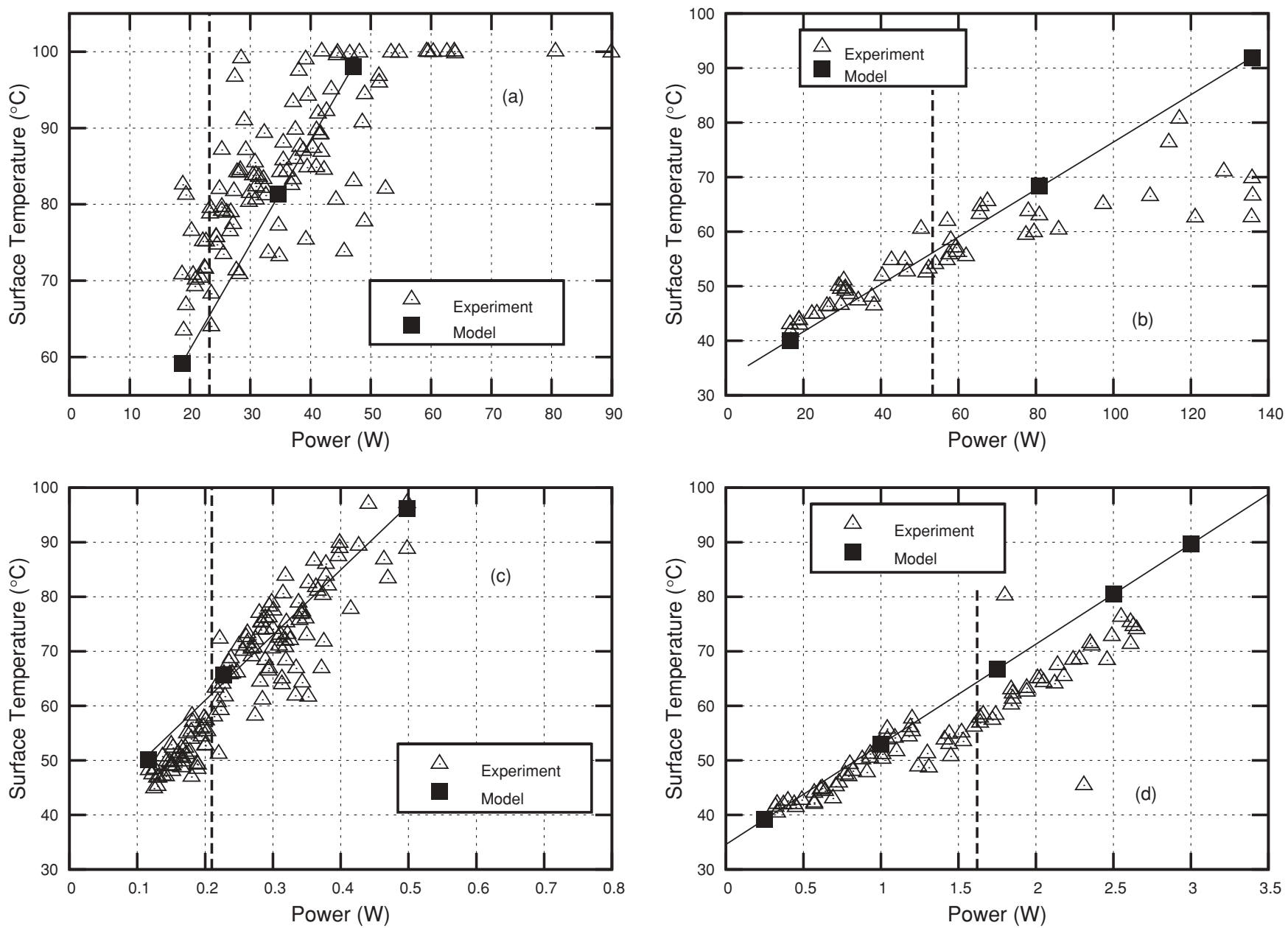

Fig. 3 Peak surface temperature during exposures as measured by thermal imagery for exposure times and Gaussian beam diameters of (a) 50 ms and $1.0 \mathrm{~cm},(\mathrm{~b}) 70 \mathrm{~ms}$ and $1.8 \mathrm{~cm}$, (c) $10 \mathrm{~s}$ and $0.48 \mathrm{~cm}$, and (d) $10 \mathrm{~s}$ and $1.8 \mathrm{~cm}$, respectively. The vertical dashed lines represent minimal visible lesion threshold in watts.

between the two wavelengths, and provide a comparison with the experimental data. The upper dotted line in the lower right demonstrates the anticipated change in threshold in going to a smaller $0.3-\mathrm{cm}\left(1 / e^{2}\right)$ beam diameter. The graph illustrates that for all but the shortest, 70-ms exposures, the model agrees well with the data, and nearly captures stated experimental uncertainties. From the data, it is clear that there is a systematic increase in damage threshold with decreasing beam diameter that is not predicted by the model. Figure 5 shows that all damage threshold curves should converge for times shorter than about one second. This discrepancy may be explained by a threshold for detectability or threshold for sufficient tissue swelling for visible observation as an endpoint.

Figure 5(b) summarizes the predicted damage threshold from 1940-nm exposures for various beam diameters as a function of exposure time. The 1-D limit is shown, demonstrating for each beam diameter the time range for which an experimental dataset would be sufficient to represent a lower bound to the damage threshold. From the figure, it is clear that the largest experimental beam diameter $(1.8 \mathrm{~cm})$ is sufficient to represent the infinite beam limit to a time of ten seconds or more. For the shorter time measurements of less than $100 \mathrm{~ms}$, it is clear that the thresholds should be in good agreement when based on peak irradiance within the beam, and when the beam is $3 \mathrm{~mm}$ or greater in diameter.

Also illustrated in Fig. 5(a) is the current exposure limit as established by the ANSI Z136.1-2007 American National Standard for Safe Use of Lasers. ${ }^{18}$ In the exposure time region of $100 \mathrm{~ms}$ to $10 \mathrm{~s}$ shown in the graph, the empirical power law trend from the safety standard mimics the estimated damage threshold, and maintains a separation of about one order of magnitude. Table 5 provides an excerpt of exposure limits for the wavelength and exposure times under consideration here.

Figure 5(b) presents an alternate view of the damage threshold exposure compared to the exposure limit. The detailed procedure within the safety standard is that the exposure limit is compared with exposure through a $3.5-\mathrm{mm}$ "limiting" aperture. ${ }^{19}$ For the analysis presented here, each beam diameter threshold power was expressed as the average exposure through a limiting aperture of $3.5 \mathrm{~mm}$. The equation for computing this average exposure is given by:

$$
E_{f}=\frac{4 \Phi_{0}}{\pi D_{L}^{2}}\left[1-\exp \left(\frac{-D_{L}^{2}}{D_{f}^{2}}\right)\right] .
$$



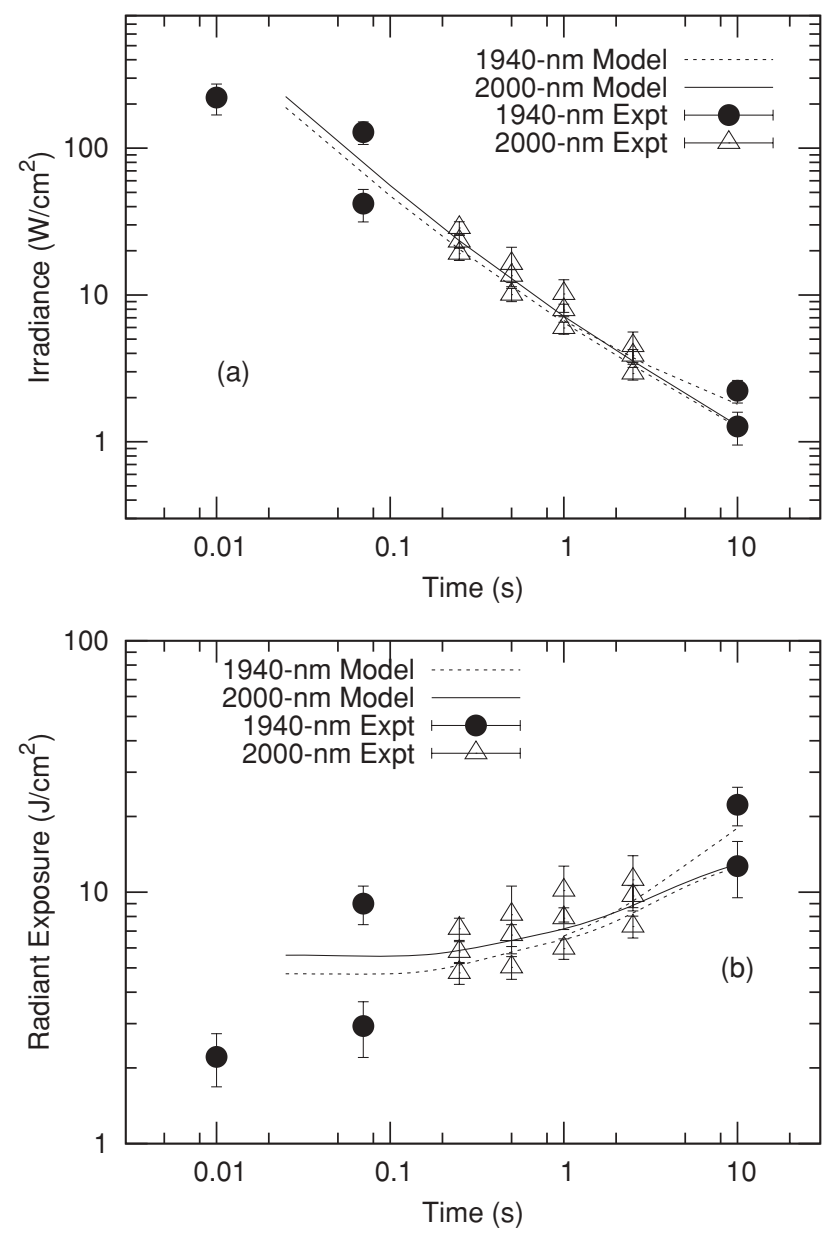

Fig. 4 Damage thresholds as functions of exposure time, expressed as (a) irradiance and (b) fluence, comparing experimental data ${ }^{2}$ at 1940 and $2000 \mathrm{~nm}$ (see text).

In Eq. (5), $D_{f}$ is the diameter of the limiting aperture (3.5 $\mathrm{mm}$ here), $D_{L}$ is the diameter of the laser beam $(1 / e$ at irradiance for a Gaussian beam), and $\Phi_{0}$ is the power of the laser. The value $E_{f}$ is the limiting-aperture-averaged exposure to be compared with the exposure limit. This procedure is designed to account for combinations of scattering and lateral

Table 5 Summary of current exposure limits for the skin in the nearto-mid infrared spectrum, from the ANSI Z136.1-2007 safety standard. Values are listed for the wavelength range in Fig. 6.

\begin{tabular}{ccccc}
\hline $\begin{array}{c}\text { Wavelength } \\
\text { (nm) }\end{array}$ & $\begin{array}{c}\text { Exposure } \\
\text { time }(\mathrm{s})\end{array}$ & $\begin{array}{c}\text { MPE } \\
{\left[\mathrm{J} / \mathrm{cm}^{2}\right]}\end{array}$ & $\begin{array}{c}\text { MPE } \\
{\left[\mathrm{W} / \mathrm{cm}^{2}\right]}\end{array}$ & $\begin{array}{c}\text { Limiting } \\
\text { aperture } \\
\text { diam }(\mathrm{mm})\end{array}$ \\
\hline 1500 to 1800 & $10^{-9}$ to 10 & 1.0 & $1.0 t^{-1}$ & 3.5 \\
1800 to 2600 & $10^{-9}$ to $10^{-3}$ & 0.1 & $0.1 t^{-1}$ & 3.5 \\
1800 to 2600 & $10^{-3}$ to 10 & $0.56 t^{0.25}$ & $0.56 t^{-0.75}$ & 3.5 \\
2600 to 4000 & $10^{-7}$ to 10 & $0.56 t^{0.25}$ & $0.56 t^{-0.75}$ & 3.5 \\
1500 to 4000 & 10 to $3 \times 10^{4}$ & $0.1 t$ & 0.1 & 3.5 \\
\hline
\end{tabular}
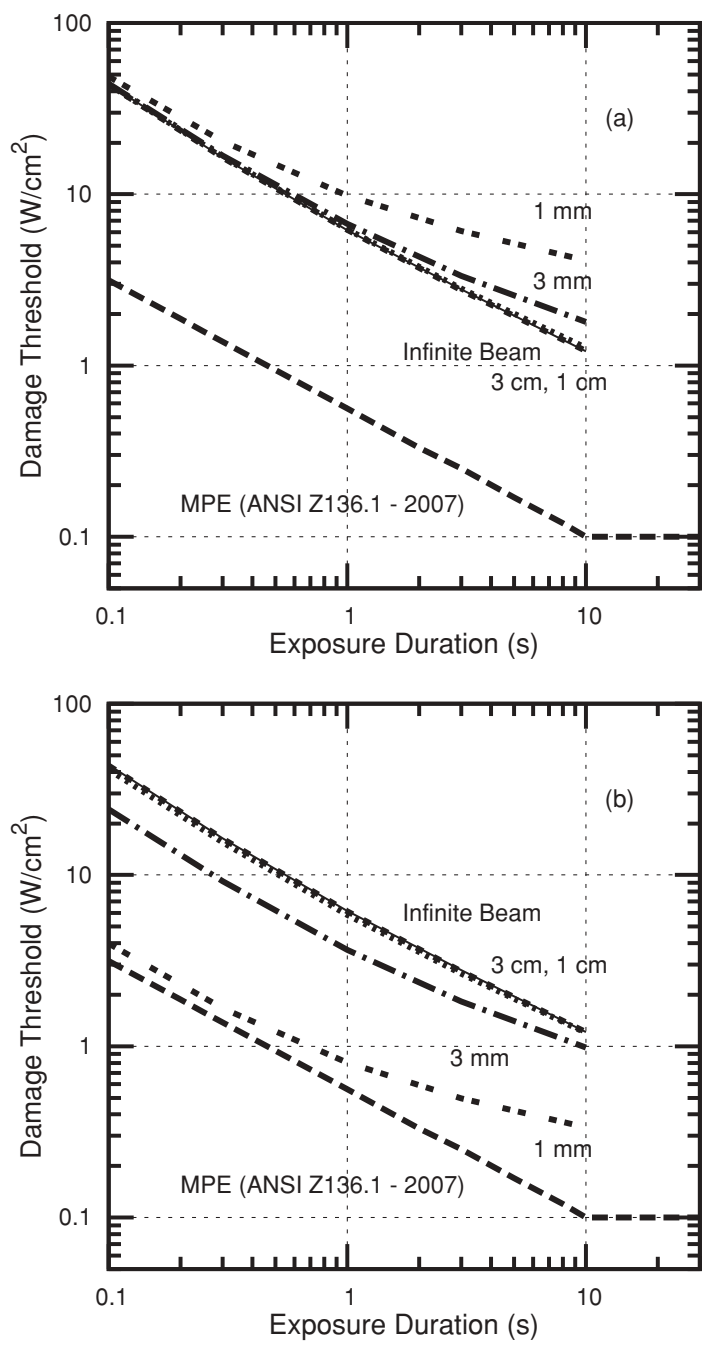

Fig. 5 Model estimates of damage threshold as (a) a function of exposure time and beam diameter, and (b) limiting aperture averaged damage threshold exposure as a function of time for $1-\mathrm{mm}, 3-\mathrm{mm}$, $1-\mathrm{cm}$, and $3-\mathrm{cm}$ beam diameter, and the infinite beam diameter (1-D radial) limit.

thermal diffusion, as seen in small beam geometries at longer times in Fig. 5. The analysis indicates that a reduced limiting aperture diameter may be warranted to avoid damage for small beam diameter exposures. The 1-mm beam diameter calculation of Fig. 5(b) shows that there is less than a factor of two in the margin of damage threshold to computed exposure when limiting apertures are invoked in the hazard assessment. However, the long term consequences of such a small area of first degree burn may be considered negligible by some experts in the laser safety community.

The second issue that must be addressed is the appropriateness of the exposure limit as a function of wavelength in the 2000-nm spectral regions. Figure 6 illustrates the dependence of absorption coefficient on wavelength for the assumed water content of epidermis and dermis. The absorption coefficient demonstrates a local maximum near $2000 \mathrm{~nm}$ and changes rapidly in regions of 1900 to $2000 \mathrm{~nm}$. A much stronger absorption band occurs near $3000 \mathrm{~nm}$ with more than two orders of magnitude of increase in absorption coefficient. Exposure limits 


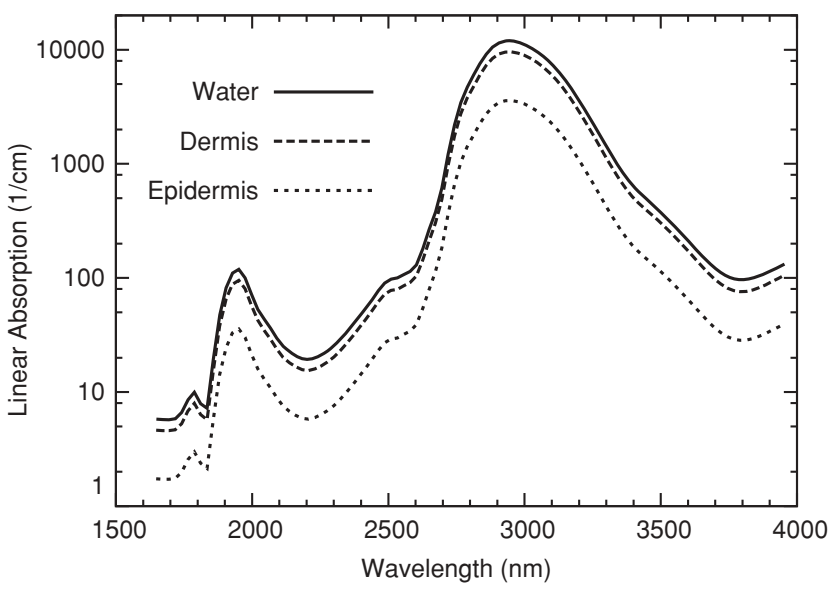

Fig. 6 Absorption coefficient for water, epidermis, and dermis skin layers as estimated from percentage water content.

in this region of the spectrum are relatively insensitive to wavelength for the separate bands of 1500 to $1800 \mathrm{~nm}$ and 1800 to $4000 \mathrm{~nm}$, each band having its own time dependence.

The model developed here allows for the evaluation of these exposure limits throughout the near-infrared region for exposure times of about $10 \mathrm{~ms}$ to $10 \mathrm{~s}$. Figure 7 provides the lowerbound estimate for damage threshold as a function of wavelength for the exposure times of $100 \mathrm{~ms}, 1 \mathrm{~s}$, and $10 \mathrm{~s}$. Along with these estimates are the prescribed exposure limits in terms of irradiance from Table 5. The analysis indicates that although optical absorption changes drastically, the threshold for damage is relatively constant, or increases with increased absorption. This constant damage threshold is due to the model constraint of damage occurring to a depth of 200 to $250 \mu \mathrm{m}$ to reach the minimally visible lesion endpoint. With increased surface

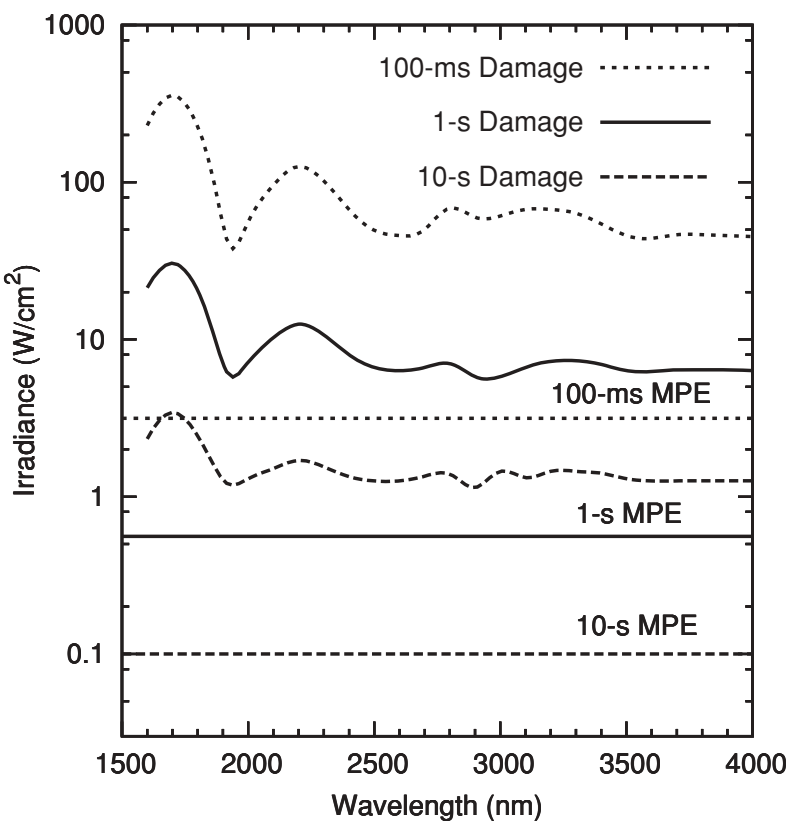

Fig. 7 Skin damage thresholds as a function of laser wavelength, estimated by a 1-D (infinite beam diameter) model and compared with current skin exposure limits. absorption, the heat must diffuse to these depths to reach the temperature to cause damage, even though the epidermal layers can reach a higher temperature than less strongly absorbing cases.

The exposure limits represented in Fig. 7 remain at the commonly seen separation of approximately one order of magnitude. Small variations are seen in separation as exposure time shortens, and are attributed to reduced axial thermal diffusion. This analysis indicates that exposure limits will maintain (for large beams) a margin of safety of at least one order of magnitude in the 3000- to 4000-nm wavelength range for exposure times of 0.1 to $10 \mathrm{~s}$.

Prior work by Lund, Edsall, and $\mathrm{Stuck}^{20}$ in the retina provides an empirical action spectrum model in which the volumetric energy density for a fixed exposure time can be used as a scaling law with wavelength. That method assumes that damage occurs at very shallow depth at threshold. As a result, the predicted damage thresholds are in some instances below currently accepted exposure limits. A comparison of results to those action spectrum models shows that the assumptions dramatically affect predicted damage thresholds. As an additional point of validation, we compare current model predictions to the experimental work compiled for cornea damage. The data compiled for the report by Dunsky and Egbert ${ }^{21}$ demonstrated that damage thresholds (for equal exposure times) are relatively constant in the cornea for absorption coefficients greater than approximately $100 \mathrm{~cm}^{-1}$. Data presented vary by less than a factor of two over the wavelength region presented in Fig. 7. As a point of comparison, the cornea data presented for $0.5-\mathrm{s}$ exposures are approximately 10 to $15 \mathrm{~W} / \mathrm{cm}^{2}$, again in good agreement with the estimates in Fig. 7.

From the analysis presented here, the conclusion is that current exposure limits maintain the commonly accepted safety margins for lasers that can be developed in these infrared bands. This conclusion applies to the exposure times of about $10 \mathrm{~ms}$ to $10 \mathrm{~s}$ examined. However, for small diameter beams, the application of current limiting apertures may result in relatively small margins of safety between damage threshold and exposure limit. In some simulations, the anticipated threshold for damage is less than $50 \%$ greater than the current exposure limits. Future studies of histology and morphology of damage can lend additional insight through validation of these modeled results.

\section{Conclusions}

Damage mechanisms at 2000 and $1940 \mathrm{~nm}$ in skin are not significantly different, as evidenced by the damage threshold levels reported here and by Chen et al. ${ }^{3,4}$ These differences in threshold level are consistent with current understanding as implemented in computational models of thermal damage. In addition, values are consistent with published values of damage at wavelengths with similar optical properties. At $1940 \mathrm{~nm}$, long exposures to skin with large spots result in a threshold value with historically accepted margins between damage threshold and exposure limits.

Computed thermal response at the skin surface serves as an intermediate validation of models when compared with experimental thermal imagery. The assumption of a requirement of a 200- to $250-\mu \mathrm{m}$ depth of damage accurately predicts the threshold of damage when compared to visible lesion endpoints. 
Overall, modeling results using the assumptions presented provide agreement commonly within about $30 \%$, with one outlier here of at most $60 \%$ over the estimate of damage threshold. The assumption reproduces the relatively wavelength-independent damage threshold trends found in the literature for strongly absorbed wavelengths.

When prescribed hazard assessment methodologies are applied through limiting aperture averaged exposures, our analysis shows that exposures will approach the damage threshold for small beams. As a result, we conclude that a reduction in limiting aperture diameters can be warranted in current exposure limits. However, skin damage associated with small-diameter beams can be viewed as negligible near threshold exposure. In addition, through the application of computer modeling and the assumptions that are consistent with experimental observations, we find that exposure limits will maintain (for large beams) a margin of safety of at least one order of magnitude in the 3000 - to $4000-\mathrm{nm}$ wavelength range for exposure times of 0.1 to $10 \mathrm{~s}$.

\section{Acknowledgments}

The authors wish to acknowledge and thank the veterinary support groups within Northrop Grumman Information Technologies, and the Air Force Research Laboratory Human Effectiveness Directorate, Directed Energy Bioeffects Division, Veterinary Sciences Branch for their expert support of these studies. Specifically, Kevin Stockton, Adam Cavazos, specialist Kassandra Heads, specialist Jessica Carr, and Aurora Shingledecker were instrumental in animal handling, test preparation, and maintenance of anesthesia during test procedures. This work was sponsored by the United States Air Force Research Laboratory, and USAF contract number F4162402-D-7003. Opinions expressed here are those of the authors and do not constitute those of the United States Air Force or Department of Defense.

\section{References}

1. J. A. Zuclich, D. J. Lund, and B. E. Stuck, "Wavelength dependence of ocular damage thresholds in the near-IR to far-IR transition region: proposed revisions to MPEs," Health Phys. 92(1), 15-23 (2007).

2. B. Chen, D. C. O'Dell, S. L. Thomsen, B. A. Rockwell, and A. J. Welch, "Porcine skin $E D_{50}$ damage thresholds for 2,000 nm laser irradiation," Lasers Surg. Med. 37, 373-381 (2005).

3. B. Chen, S. L. Thomsen, R. J. Thomas, and A. J. Welch, "Modeling thermal damage in skin from 2000-nm laser irradiation," J. Biomed. Opt. 11, 15 (2006).
4. B. Chen, S. Thomsen, R. J. Thomas, J. Oliver, and A. J. Welch, "Histological and modeling study of skin thermal injury to 2.0 micron laser irradiation," Lasers Surg. Med. 40, 358-370 (2007).

5. T. A. Eggleston, W. P. Roach, M. A. Mitchell, K. Smith, D. Oler, and T. E. Johnson, "Comparison of two porcine (sus scrofa domestica) skin models for in vivo near-infrared laser exposure," Compar. Med. 50(4), 391-397 (2000).

6. A. Welch and M. van Gemert, Optical-Thermal Response of LaserIrradiated Tissue, Plenum Press, New York (1995).

7. M. H. Niemz, "Laser tissue interactions, fundamentals and applications," Biological and Medical Physics, Biomedical Engineering, 3rd ed., Springer-Verlag, New York (2004).

8. M. A. Mainster, T. J. White, J. H. Tips, and P. W. Wilson, "Transient thermal behavior in biological systems," Bull. Math Biophys. 32, 303314 (1970).

9. A. N. Takata, L. Zaneveld, and W. Richter, "Laser-induced thermal damage of skin," Tech. Rep., IIT Research Institute, Chicago, IL (1977).

10. R. Birngruber, "Experimental studies of laser thermal retinal injury," Health Phys. 44, 14 (1983).

11. R. A. London, M. E. Glinsky, D. S. Bailey, G. B. Zimmerman, and D. C. Eder, "Laser-tissue interaction modeling with the LATIS computer program," Tech. Rep. UCRL-LR-105821-96-3, Lawrence Livermore National Laboratory, Livermore, CA (1996).

12. D. J. Maitland, D. C. Eder, R. A. London, M. E. Glinsky, and B. A. Soltz, "Dynamic simulations of tissue welding," Proc. SPIE 2671, 234-242 (1996).

13. J. H. Torres and M. Motamedi, "Experimental evaluation of mathematical models for predicting the thermal reaponse of tissue to laser irradiation," Appl. Opt. 32(13), 597-606 (1993).

14. D. C. Gaylor, "Physical mechanisms of cellular injury in electrical trauma," PhD Thesis, Massachusetts Institute of Technology (1989).

15. M. L. Denton, M. S. Foltz, G. Noojin, L. E. Estlack, R. J. Thomas, and B. A. Rockwell, "Determination of threshold average temperature for cell death in an in vitro retinal model using thermography," Proc. SPIE 7175(1), 71750G (2009).

16. D. J. Finney, Probit Analysis, Cambridge University Press, Boston, MA (1971).

17. A. M. Stoll and L. C. Greene, "Relationship between pain and tissue damage due to thermal irradiation," J. Appl. Physiol. 14(3), 372-382 (1959).

18. American National Standards Institute, ANSI Z136.1 American National Standard for Safe Use of Lasers, Laser Institute of America, Orlando, FL (2007).

19. R. J. Thomas, B. A. Rockwell, W. J. Marshall, R. C. Aldrich, M. F. Gorschboth, and S. A. Zimmerman, "A procedure for the estimation of intrabeam hazard distances and optical density requirements under the ANSI Z136.1-2000 standard," J. Laser Appl. 16(3), 167-177 (2004).

20. D. J. Lund, P. Edsall, and B. E. Stuck, "Ocular hazards of q-switched near-infrared lasers," Proc. SPIE 4953, 85-90 (2003).

21. I. L. Dunsky and D. E. Egbert, "Corneal damage thresholds for hydrogen flouride and deuterium fluoride chemical lasers," Tech. Rep. SAM TR 73-51, USAF School of Aerospace Medicine (1973).

22. F. F. Henriques, "Studies of thermal injury v: the predictability and the significance of thermally induced rate processes leading to irreversible epidermal injury," Arch. Pathol. 43, 489-502 (1947). 